\title{
Editorials
}

\section{Is primary care research important and relevant to GPs?}

\section{INTRODUCTION}

Insufficient investment in primary care services, even in high-income countries, is challenging health systems, for example, through pressures on the primary care workforce from rising workloads, ageing practitioner profiles, and declining interest in primary care careers from young practitioners. Failure to invest in research to guide better practice, raising quality and reducing variations in care, may be a further risk. But is primary care research really that important?

\section{IMPORTANCE AND DOMINANCE OF BASIC SCIENCE MEDICAL RESEARCH}

Most biomedical research has historically been centred on basic science and the pursuit of discovery, for example, on the mechanisms of disease and their amelioration. Until latterly, the application of this science was seen merely as a professional responsibility - why don't practitioners just get on and apply the important findings? But such implementation delays may be more to do with incomplete evidence than clinical inertia, hence one argument for more applied research evidence to inform discovery science.

A century of serial multi-billion-dollar investments in basic and discovery science generated the interventions we use today. But this investment is being challenged by the dawning recognition by health systems that they need a better evidence base to inform the structure and provision of care, and how to apply the findings of much of this extraordinary biomedical science. Despite basic research consuming most of the global health research investment, much of health care remains empirical and nonevidence based. And, even when evidence gives a clear directive on what to do, slow translation into clinical practice may be as often due to uncertainties as to how to apply the data than on professional inertia.

Belated recognition of such barriers to implementation has led to the introduction of a more applied clinical research focus in many countries with developed healthcare systems, such as the NHS, that complements basic science and discovery research. First came the science around evidence-based medicine (EBM), for 20 years developing and employing better methods of synthesising and presenting the totality of quality evidence

"Primary care evidence has regularly impacted on informing international clinical guidelines...

and thereby reducing uncertainty. Latterly an investment in applied health research, and the capacity to better deliver this, has gathered impetus internationally, for example, via the main driver for such applied research in the UK, the National Institute for Health Research (NIHR).

Primary care and primary care academics have steadily contributed to many aspects of health research, but it has been particularly important in applied research at the structural and inspirational levels.

\section{DO WE NEED PRIMARY CARE RESEARCH?}

But, is research based within primary care important? Given that most patient contacts originate and end in primary care in most developed health systems, the necessity to research more within primary care is obvious. The full spectrum of disease is represented, the long trajectory of disease is discoverable, and the patient subjects are representative of the total population and demonstrate the full range of behaviours. The traditional model of researching more extreme phenotypes, represented in hospital-recruited cohorts, remains important, and efficient, for early research and estimating the likely best effects of interventions, partly because patients triaged to hospital will mostly experience higher and earlier event rates. Care in specialist settings also needs to be based on research conducted in specialist settings. However, the corollary should be selfevident - care in the community should be based on evidence from community populations, whether for diagnostic test performance and thresholds or for therapeutic interventions.

Failure to provide such 'context' evidence has historically contributed to confusion and delay in applying promising evidence to the clinical care of the masses, hence 'my patients aren't like those included in that trial'. Such protestations should diminish with applied research refining answers to 'what to do' with showing 'how to do it'.

One important rider to evidence-based practice is the risk of nihilism - science is provisional and rarely absolute, and we can't wait indefinitely before acting. Until we have entirely predictable personalised medicine (a generation off, if ever), mostly we will have to apply best evidence to the population and accept that some may not benefit or may even be harmed. Patients, I think, understand these realities better than physicians do sometimes and deserve neutral presentation of uncertainty, even when they respond by saying, 'But what would you do, doctor?' There is, of course, also the important risk of over-diagnosis and over-treatment, more prominently highlighted than clinical inertia, which mandates ensuring that the limitations of data are recognised.

Health science therefore needs evidence derived from the populations in which that evidence is to be applied, so that research in primary care is scientifically essential. There are also some important practical benefits of such an approach: it is increasingly difficult to recruit to landmark trials, especially with active comparators, without recruiting in primary care, and doctors involved in research are more likely early adopters of technologies found to be effective - an efficient implementation strategy.

\section{HISTORIC CONTRIBUTIONS OF PRIMARY CARE ACADEMICS TO CLINICAL SCIENCE}

Yet, having argued that research based in primary care is important, whether we need primary care academics to conceptualise and deliver some of this research deserves answering. The influence of primary care researchers is not a modern phenomenon. The origins of high-quality clinical research had primary care champions: Edward Jenner, who in the $18^{\text {th }}$ century observed associations with smallpox and trialled a candidate cowpox vaccine, thus founding the science of immunisation, was a West Country family doctor; John Snow, a mid-19th-century founding father of epidemiology, was also a family doctor.

Thirty years ago, important research by 
academic GPs explained what makes better consultations ${ }^{1}$ and provided observations on the inadequacies of health care $^{2}$ that helped spawn EBM. ${ }^{3}$ Primary care researchers, clinical and methodological, are strongly represented in advancing EBM internationally and have become essential contributors to more reliable methods of generating evidence-based guidelines, ${ }^{4}$ a key mechanism used by health systems to define the expected standards of care expected of practitioners working in the system.

\section{RECENT CONTRIBUTIONS OF PRIMARY CARE ACADEMICS TO CLINICAL SCIENCE}

The impact of primary care academics has accelerated in the past 20 years, and across many major clinical areas. There is notable health services research on surrogate measures for quality of care, evidence on whether pay for performance works, ${ }^{5}$ and even what happens when incentives stop, ${ }^{6}$ all providing key evidence for more evidencebased policy development. A major current deficit in most health systems is trial-anderror planning - risky and expensive given it is difficult to de-commission services once they are provided to the public

In terms of evidence for clinical practice there are major advances in utilising diagnostics more effectively and safely in primary care, providing the key missing data for better implementation of evidence from specialist settings, such as the necessary performance of validated disease risk scores; ${ }^{7}$ the most costeffective methods of diagnosing and then managing hypertension; ${ }^{8}$ and thresholds for biomarkers, such as natriuretic peptides in symptomatic patients, ${ }^{9}$ all helping to triage at-risk populations more efficiently. Such primary care researchers, in partnership with the practices or primary care research networks that conduct the research, are also contributing to the evidence for screening or early detection of major impact disorders, such as diabetes, ${ }^{10}$ atrial fibrillation, ${ }^{11}$ and heart failure.

In clinical management, in addition to major work in major long-term disorders, primary care academics have made major contributions to infection research including antibiotic conservation, ${ }^{12}$ and to acute problems such as Bell's palsy. ${ }^{13}$ The long-term primary care interest in health services research has continued, but with higher-quality study designs, reporting on what makes more effective consultations, or how to configure primary care, ${ }^{14}$ or focus on major social issues.
A tradition of primary care academics in public health research has also continued, for example, highlighting the emerging importance of multimorbidity in our increasingly ageing populations, ${ }^{15}$ with the ongoing challenges of determining the most impactful disease combinations and predicting the most adverse pathways for testing interventional strategies. It is possible that deep genotypic categorisation may ultimately predict personal health trajectories, but there will be decades of needing better phenotyping and predictive models.

In conclusion, the importance and practice relevance of more research based in primary care is clear, as is the essential role for academic primary care and its partnerships with practices. Primary care evidence has regularly impacted on informing international clinical guidelines, which is itself a useful surrogate for relevance and impact. The rate of such research has accelerated in the past 20 years and the quality and complexity has increased. Health systems should highlight the importance of primary care research to help deliver and guide priorities, and invest in the academics and research practice networks that provide the evidence.

\section{REFERENCES}

1. Howie JG, Heaney DJ, Maxwell M, et al. Quality of general practice consultations: cross sectional survey. BMJ 1999; 319(7212): 738-743.

2. Hart JT. The inverse care law. Lancet 1971; 1(7696): 405-412

3. Sackett DL, Rosenberg WM, Gray JA, et al. Evidence based medicine: what it is and what it isn't. BMJ 1996; 312(7023): 71-72

4. Heneghan C, Alonso-Coello P, GarciaAlamino JM, et al. Self-monitoring of oral anticoagulation: a systematic review and metaanalysis. Lancet 2006; 367(9508): 404-411.

5. Sutton M, Nikolova $S$, Boaden $R$, et al. Reduced mortality with hospital pay for performance in England. N Engl J Med 2012 367(19): 1821-1828.

6. Minchin M, Roland M, Richardson J, et al. Quality of care in the United Kingdom after removal of financial incentives. N Engl J Med 2018; 379(10): 948-957

7. Hippisley-Cox J, Coupland C, Vinogradova $Y$, et al. Predicting cardiovascular risk in England and Wales: prospective derivation and validation of QRISK2. BMJ 2008; 336(7659): 1475-1482.

8. Lovibond K, Jowett S, Barton P, et al. Costeffectiveness of options for the diagnosis of high blood pressure in primary care: a modelling study. Lancet 2011; 378(9798): 1219-1230.

9. Hobbs FD, Davis RC, Roalfe AK, et al. Reliability of $\mathrm{N}$-terminal pro-brain natriuretic peptide

\section{ADDRESS FOR CORRESPONDENCE}

\section{Richard Hobbs}

Nuffield Department of Primary Care Health

Sciences, Radcliffe Primary Care Building, Radcliffe Observatory Quarter, Oxford OX2 6GG, UK.

\section{Email: richard.hobbsaphc.ox.ac.uk}

\section{Richard Hobbs,}

Professor; Head, Nuffield Department of Primary Care Health Sciences, University of Oxford, Oxford; Director, National Institute for Health Research English School for Primary Care Research.

\section{Funding}

The author has received part-funding from the National Institute for Health Research (NIHR) School for Primary Care Research, the NIHR Collaboration for Leadership in Health Research and Care (CLARHC) Oxford, the NIHR Oxford Biomedical Research Centre (BRC, UHT), and the NIHR Oxford Medtech and In-Vitro Diagnostics Co-operative (MIC)

\section{Provenance}

Freely submitted; externally peer reviewed.

\section{Competing interests}

The views expressed in this editorial are those of the author, and not necessarily those of the NIHR.

DOI: https://doi.org/10.3399/bjgp19X705149

assay in diagnosis of heart failure: cohort study in representative and high risk community populations. BMJ 2002; 324(7352): 1498

10. Griffin SJ, Little PS, Hales CN, et al. Diabetes risk score: towards earlier detection of Type 2 diabetes in general practice. Diabetes Metab Res Rev 2000; 16(3): 164-171.

11. Hobbs FD, Fitzmaurice DA, Mant J, et al. A randomised controlled trial and costeffectiveness study of systematic screening (targeted and total population screening) versus routine practice for the detection of atrial fibrillation in people aged 65 and over. The SAFE study. Health Technol Assess 2005; 9(40): iii-iv, ix-x, 1-74.

12. Little P, Moore M, Kelly J, et al. Delayed antibiotic prescribing strategies for respiratory tract infections in primary care: pragmatic, factorial, randomised controlled trial. BMJ 2014; 348: g1606

13. Sullivan FM, Swan IR, Donnan PT, et al. Early treatment with prednisolone or acyclovir in Bell's palsy. N Engl J Med 2007; 357(16): 1598-1607.

14. Horrocks S, Anderson E, Salisbury C. Systematic review of whether nurse practitioners working in primary care can provide equivalent care to doctors. BMJ 2002; 324(7341): 819-823.

15. Barnett K, Mercer SW, Norbury M, et al. Epidemiology of multimorbidity and implications for health care, research, and medical education: a cross-sectional study. Lancet 2012; 380(9836): 37-43. 